\title{
Registro de Acrocinus longimanus (Linnaeus, 1758) (Coleoptera: Cerambycidae) en Sucre, Caribe Colombiano
}

\section{Record of Acrocinus longimanus (Linnaeus, 1758) (Coleoptera: Cerambycidae) in Sucre, Colombian Caribbean}

\author{
Fuentes-Mario, José A. ${ }^{1 *}$ Biol.; Salcedo-Rivera, Gerson A. ${ }^{1}$ Biol.
}

${ }^{1}$ Universidad de Sucre, Grupo de Investigación en Biodiversidad Tropical. Sincelejo - Colombia.

\section{KEYWORDS:}

Acrocinus longimanus; urban area; Tropical Dry Forest; Caribbean; Colombia.

\section{ABSTRACT}

The presence of Acrocinus longimanus (Linnaeus, 1758) (Coleoptera: Cerambycidae) is documented in the department of Sucre - Colombia, with special reference for the urban area of the municipality of Toluviejo, adjacent to a Tropical Dry Forest formation.

\section{PALABRAS CLAVE:}

Acrocinus longimanus; área urbana; Bosque Seco Tropical; Caribe; Colombia.

\section{RESUMEN}

Se documenta la presencia de Acrocinus longimanus (Linnaeus, 1758) (Coleoptera: Cerambycidae) en el departamento de Sucre - Colombia, con especial referencia para el área urbana del municipio de Toluviejo, aledaño a una formación de Bosque Seco Tropical. 
Acrocinus longimanus (Linnaeus, 1758), conocido popularmente como "escarabajo arlequín", es un coleóptero perteneciente a la familia Cerambycidae, cuyo rango de distribución conocido va desde el sur de México hasta Brasil y el norte de Argentina (ZEH et al., 1992; DOS SANTOS y PEREIRA-COLAVITE, 2017), incluyendo también la Guyana Francesa y Trinidad y Tobago (RANDEEM, 2015), países donde usualmente habita áreas boscosas no perturbadas, siendo distinguido a menudo en bosques lluviosos de la Amazonia (RANDEEM, 2015).

Sobre su biología se destaca que es una especie con un gran porte, ya que los machos pueden llegar a presentar longitudes de las patas anteriores hasta de $150 \mathrm{~mm}$ (DOS SANTOS y PEREIRA-COLAVITE, 2017), aunque el dimorfismo sexual es más conspicuo en la forma de la tibia anterior, siendo la de los machos curvada, mientras que la de las hembras es casi recta (ZEH et al., 1992). Las hembras ponen los huevos en árboles caídos ricos en savia, que posteriormente las larvas aprovechan (ZEH et al., 1992); y los adultos son principalmente herbívoros (RANDEEM, 2015), encontrándose en árboles de la familia Moraceae y Apocynaceae, que son usados, además, para efectos de reproducción, oviposición y desarrollo de las larvas (DOS SANTOS y PEREIRA-COLAVITE, 2017); sin embargo, también son detritívoros, teniéndose datos acerca de su sobrevivencia exclusiva a partir de excremento de otros animales (RANDEEM, 2015). En Brasil esta especie se ha identificado como plaga de "painera", "eucalipto" y "figueira" (DOS SANTOS y PEREIRA-COLAVITE, 2017), y en El Salvador tiene un impacto barrenador sobre Brosimum alicastrum (VELADO-CANO y PABLO-CEA, 2012). Además, los individuos tienen actividad diurna, pero también son atraídos por las luces artificiales en la noche (RANDEEM, 2015); y, aunque es una especie solitaria (RANDEEM, 2015), se ha encontrado que exhibe foresis con otros organismos, como el seudoescorpión Cordylochernes scorpioides (COLORADO y TORRES BEJARANO, 2016).

La Unión Internacional para la Conservación de la Naturaleza (UICN) lista a A. longimanus como vulnerable (RANDEEM, 2015). Es necesario enfatizar que, según RANDEEM (2015), una de sus amenazas principales es la extensión de la deforestación de su hábitat natural causada por el hombre, que produce una reducción en el tiempo de reproducción y también una interrupción de las extensas etapas larvales, sin lograrse su finalización; no obstante, este autor comentó que la especie también sufre deterioro poblacional por efectos del mascotismo y el coleccionismo ilegal.

En Colombia se tienen actualmente 44 registros en el Sistema de Información sobre Biodiversidad de
Colombia - SiB Colombia (SIB COLOMBIA, 2018), resaltando su presencia en los departamentos de Amazonas, Antioquia, Chocó, Cundinamarca, Magdalena, Meta, Santander, Tolima y Valle del Cauca; empero, en esta nota se presenta que $A$. longimanus fue avistado durante la tarde del 27 de septiembre de 2014 en el área urbana del municipio de Toluviejo, Sucre - Colombia $\left(9^{\circ} 26^{\prime} 59,124^{\prime \prime} \mathrm{N}-75^{\circ} 26^{\prime} 30,618^{\prime \prime} \mathrm{W}\right)$, por uno de los autores (J.A. Fuentes-Mario), quien fotografió a un individuo macho vivo (Figura 1) que había sido encontrado por un habitante de la zona durante sus labores en una institución de educación media localizada en el casco urbano (Figura 1), pero quien decidió no donarlo a ninguna colección de referencia, pues, su intención era liberarlo en un área boscosa cercana, por lo que fue imposible tomar las respectivas medidas morfométricas, aunque el individuo fue identificado a través de las fotografías, siguiendo las anotaciones e ilustraciones presentadas en el trabajo de ZEH et al. (1992).

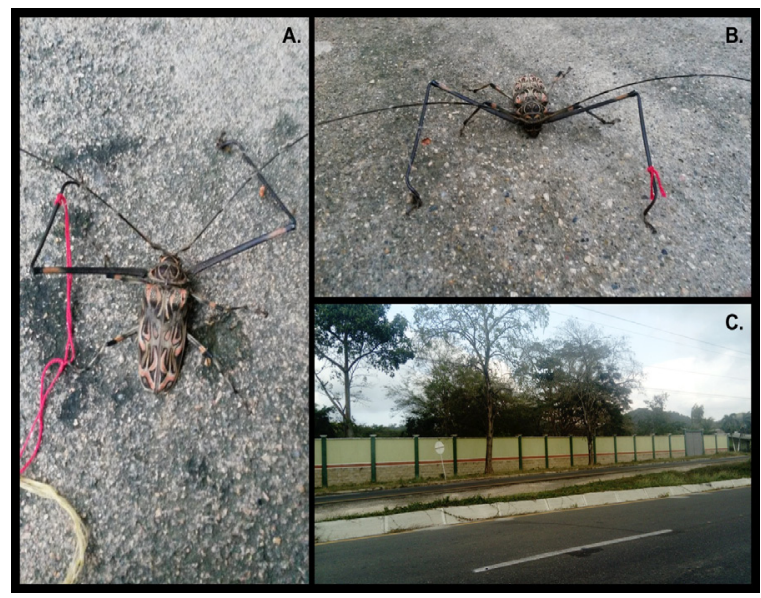

Figura 1. A. y B. Vistas dorsal y dorso-anterior del individuo macho vivo de Acrocinus longimanus capturado el día 27/09/2014 en el área urbana del municipio de Toluviejo, Sucre - Colombia. C. Área verde localizado en el interior de la institución de educación media donde se capturó el espécimen; además de este paisaje urbano, obsérvese la formación montañosa al fondo, correspondiente a un fragmento de Bosque Seco Tropical.

Para corroborar y validar la información de la presencia, entre septiembre y noviembre de 2017 se decidió visitar varias zonas rurales de Toluviejo en busca de colectar ejemplares, o en su defecto, encontrar evidencias de $A$. longimanus por efecto barrenador en árboles de algunas áreas de bosque; sin embargo, no se obtuvieron resultados, por lo que, como tercera medida, se optó por presentar fotografías de la especie y realizar entrevistas a pobladores del municipio (tanto en el área urbana como en el área rural), encontrándose que, de 20 entrevistados, 12 comentaron que, por lo menos, en alguna ocasión de su vida han visto a un individuo de $A$. 
longimanus, de la cual refirieron que, en las épocas de lluvia, muy esporádicamente puede vérsele durante las primeras horas de la mañana, pernoctando en árboles de áreas boscosas de los corregimientos La Piche, La Esperanza y Varsovia, correspondientes a Bosque Seco Tropical; aunque también afirmaron que nunca lo han visto en el casco urbano o cercanías del mismo.

Según DOS SANTOS y PEREIRA-COLAVITE (2017), el conocimiento sobre la biología y la ecología de la especie es escaso; no obstante, estos mismos autores presentaron un trabajo en el que se registró la presencia de $A$. longimanus en un área de refugio en el perímetro urbano de Caruaru, Pernambuco Brasil, cuyas características ambientales principales son clima semiárido caliente y vegetación original de catinga abierta (Bosque Tropical Estacionalmente Seco) (DOS SANTOS y PEREIRA-COLAVITE, 2017); de esta forma, el presente trabajo también es un nuevo aporte del registro de la ocurrencia de la especie en zonas urbanas aledañas a áreas de Bosque Seco Tropical.

La presencia de coleópteros de gran tamaño como este en áreas antropizadas y fuera de su hábitat natural puede explicarse, en parte, porque son atraídos por las luces artificiales (RATCLIFFE y MORÓN, 2005; GASCA-ÁLVAREZ e HIGUERA, 2007), lo que concuerda con la vasta iluminación eléctrica del lugar donde se dio la captura; sin embargo, las áreas verdes encontradas en los paisajes urbanos, que, además, podrían convertirse en "nuevos ecosistemas", también pueden explicar la ocurrencia de especies como $A$. longimanus en estos lugares (ERNSTSON et al., 2010; DOS SANTOS y PEREIRA-COLAVITE, 2017), al llegar a ser refugios importantes para estos insectos (LUZZI et al., 2016), dado que, aunque en muchos casos el proceso de urbanización y la expansión de la frontera agrícola conllevan a la pérdida de diversidad biológica (MCKINNEY, 2008), en otros, las áreas urbanas y densamente pobladas pueden contener recursos beneficiosos para los insectos (ZANETTE et al., 2005; FETRIDGE et al., 2008; PEREIRA-PEIXOTO et al., 2014), por lo que también deben ser considerados como paisajes de vital importancia para conservar la diversidad biológica (DOS SANTOS y PEREIRACOLAVITE, 2017).

Agradecimientos: Nuestro total agradecimiento va dirigido a la señora Iris Margot Álvarez Pestana (Q.E.P.D.), por permitirnos la oportunidad de observar y fotografiar al individuo de $A$. longimanus, que ella misma capturó por curiosidad y posteriormente liberó. Lamentablemente, la señora Iris falleció el martes 20 de febrero de 2018 a sus 64 años; no obstante, dedicamos esta contribución a su memoria.

\section{REFERENCIAS}

COLORADO, G.J.; TORRES-BEJARANO, A.M. 2016. New geographic distribution record of the phoretic association between the cerambycid beetle Acrocinus longimanus and the pseudoscorpion Cordylochernes scorpioides in the Colombian Amazonia. Mundo Amazónico 7 (1-2):111-114.

DOS SANTOS, W.E.; PEREIRA-COLAVITE, A. 2017. Ocorrência de Acrocinus longimanus (Linnaeus) (Coleoptera: Cerambycidae) em área urbana, Nordeste do Brasil. EntomoBrasilis 10 (1):57-59.

ERNSTSON, H.; BARTHEL, S.; ANDERSSON, E.; BORGSTRÖM, S.T. 2010. Scale-c brokers and network governance of urban ecosystem services: the case of Stockholm. Ecology and Society 15 (4):28.

FETRIDGE, E.D.; ASCHER, J.S.; LANGELLOTTO, G.A. 2008. The bee fauna of residential gardens in a suburb of New York City (Hymenoptera: Apoidea). Annals of the Entomological Society of America 101:1067-1077.

GASCA-ÁLVAREZ, H.J.; HIGUERA, D. 2007. Observaciones preliminares de la abundancia, hábitats y actividades de extracción del escarabajo elefante Megasoma mars Reiche, 1852 en zonas aledañas a la ciudad de Leticia (Amazonas-Colombia). Artículos de los informes - Becas IEA 2003-2007. Bogotá, D.C.

LUZZI, J.R.; MACIEL, T.T.; BARBOSA, B.C. 2016. Ocorrência de Megasoma gyas gyas (Herbst, 1785) (Coleoptera: Scarabaeidae) em perímetro urbano. Entomotropica 31:60-63.

MCKINNEY, M.L. 2008. Effects of urbanization on species richness: a review of plants and animals. Urban Ecosystems 11:161-176. 
PEREIRA-PEIXOTO, M.H.; PUFAL, G.; MARTINS, C.F.; KLEIN, A.M. 2014. Spillover of trap-nesting bees and wasps in an urban-rural interface. Journal of Insect Conservation 18:815-826.

RAMDEEN, T. 2015. Acrocinus longimanus (Harlequin Beetle) - The Online Guide to the Animals of Trinidad and Tobago. Disponible en: URL: https://sta.uwi.edu/fst/lifesciences/sites/default/files/lifesciences/images/Acrocinus longimanus- Beetle, Harlequin.pdf. Consultado: 22-02-2018.

RATCLIFFE, B.C.; MORÓN, M.A. 2005. Larval descriptions of eight species of Megasoma Kirby (Coleoptera: Scarabaeidae: Dynastinae) with a key for identification and notes on biology. Coleopterists Bulletin 59:91-126.

SIB COLOMBIA. 2018. Datos de registros biológicos colombianos de Acrocinus longimanus. Disponible en: URL: http://data.sibcolombia.net/. Consultado: 22-02-2018.

VELADO-CANO, M.A.; PABLO-CEA, J.D. 2012. Impacto de escarabajo barrenador (Acrocinus longimanus) sobre los árboles de ojushte (Brosimum alicastrum) y el efecto indirecto sobre la población de monos araña (Ateles geoffroyi) en el Área Natural Protegida Chaguantique, Puerto EI Triunfo, Usulután, El Salvador. Reporte final de horas sociales. Universidad de El Salvador, Facultad de Ciencias Naturales y Matemátic., San Salvador.

ZANETTE, L.R.; MARTINS, R.P.; RIBEIRO, S.P. 2005. Effects of urbanization on Neotropical wasp and bee assemblages in a Brazilian metropolis. Landscape and Urban Planning 71:105-121.

ZEH, D.W.; ZEH, J.A.; TAVAKILIAN, G. 1992. Sexual selection and sexual dimorphism in the harlequin beetle Acrocinus longimanus. Biotropica 24 (1):86-96. 